\title{
L'Eveil aux langues come motore di ricerca per un progetto di multiculturalità
}

\section{Marina Garbolino Riva}

\section{(2) OpenEdition \\ 12 Journals}

Edizione digitale

URL: http://journals.openedition.org/esp/2966

DOI: 10.4000/esp.2966

ISSN: 2532-0319

Editore

Centre d'Information sur l'Éducation Bilingue et Plurilingue

\section{Edizione cartacea}

Data di pubblicazione: 1 décembre 2018

Paginazione: 23-32

ISSN: 1127-266X

Notizia bibliografica digitale

Marina Garbolino Riva, « L'Eveil aux langues come motore di ricerca per un progetto di

multiculturalità », Éducation et sociétés plurilingues [Online], 45 | 2018, Messo online il 01 mars 2020,

consultato il 04 décembre 2020. URL : http://journals.openedition.org/esp/2966 ; DOI : https://doi.org/ 10.4000/esp.2966 


\section{L'EVEIL AUX LANGUES COME MOTORE DI RICERGA PER UN PROGETTO DI MULTICULTURALITÀ}

\section{Marina GaRbolino RIVA}

Organiser un projet d'éducation bilingue ou plurilingue (éveil aux langues) à l'intérieur de l'école maternelle, en mettant en place aussi bien l'utilisation d'instruments didactiques novateurs que la coopération de parents d'une culture différente de l'italienne: voilà l'engagement qui a caractérisé notre activité didactique pendant toute l'année scolaire 2017/2018 et que nous souhaitons raconter et documenter dans ces pages au contenu fortement expérimental.

Mots-clés: éveil aux langues, approche plurilingue, langues familiales, dimension monde, parents en classe.

Organizing a project of bilingual and multilingual education (language awareness) inside a nursery school, by applying innovative teaching methods and inviting the cooperation of culturally diverse parents is what characterizes the didactic approach implemented throughout the 2017/2018 school year that we wish to document and explain in the following, decidely experimental article.

Parole-chiave: language awareness, multilingual approach, family languages, worldwide dimension, parents in the class.

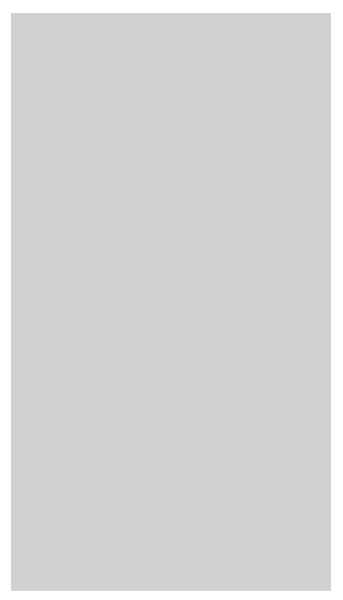

Tl sistema scolastico valdostano non solo prevede l'insegnamento della lingua francese già a partire dalla scuola dell'infanzia, Ler un numero di ore pari a quelle riservate alla lingua italiana, ma si apre sempre di più ad una prospettiva di educazione plurilingue, anche per dare una risposta di efficace integrazione ai bambini stranieri sempre più presenti nelle nostre scuole. Il fenomeno migratorio, così rapido e complesso, chiede infatti alla scuola interventi meditati e culturalmente fondati, sperimentazioni didattiche ed educative che diventino esperienza e che siano sostenute e confortate dalla ricerca e dalla riflessione. $\grave{E}$ in questo contesto che la nostra scuola dell'infanzia ha fatto proprio l'approccio di insegnamento delle lingue denominato éveil aux langues, utilizzandolo come motore di ricerca per un progetto di multiculturalità in grado di mettere a confronto, valorizzare e dare pari dignità alle diverse culture presenti al suo interno. Questo investimento ha rappresentato una sfida pedagogica concreta, che 


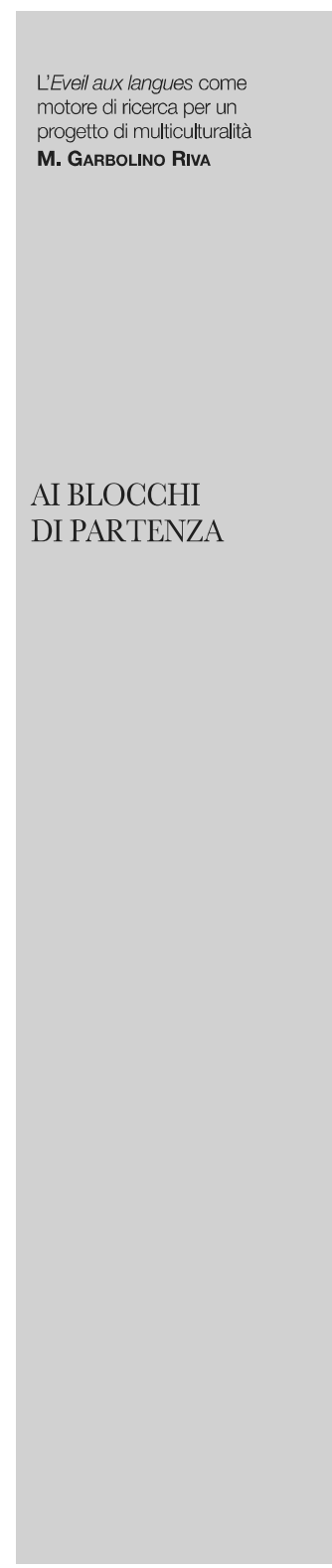

abbiamo voluto cogliere per dare un indirizzo preciso alla costruzione dell'identità di una istituzione che si sta modificando, sia per i nuovi e differenti ingressi, sia per le mutate esigenze dei bambini e delle loro famiglie. Nella scuola dell'infanzia non è possibile ipotizzare porte chiuse; essa è riconosciuta come l'ambito educativo più accogliente, capace di essere, ancora prima che luogo di istruzione, un luogo dove avvengono nuovi processi di socializzazione e di integrazione. In tale contesto la progettazione collegiale del curricolo bi-plurilingue assume un ruolo fondamentale, specialmente se rafforzata da attività di formazione per le insegnanti e dalla predisposizione di materiali didattici innovativi c performanti

La nostra scuola dell'infanzia è paritaria, ha sede nella città di Aosta e accoglie 69 bambini, suddivisi in tre sezioni miste per età. Il gruppo docente è costituito da cinque insegnanti, di cui una di sostegno, e da una coordinatrice delle attività didattico-educative. Nella programmazione annuale 2016/2017 abbiamo ridefinito gli obiettivi riguardanti l'area linguistica, allo scopo di aiutare i bambini a raggiungere un buon livello di comprensione e di utilizzo della lingua francese, e un discreto risultato nell'approccio alla lingua inglese. Per quest'ultima i destinatari privilegiati erano $\mathrm{i}$ bambini dell'ultimo anno, in vista della loro scolarizzazione; tuttavia ci siamo rese conto della capacità dei bambini più piccoli di impadronirsi delle parole udite nei racconti e di riutilizzarle, sia nei tempi di gioco libero, sia in quelli organizzati dalle insegnanti. Per l'apprendimento della lingua francese confidavamo nell'efficacia dei materiali usati in questi anni, che avevamo costantemente rinnovato sulla base di esperienze soddisfacenti e che erano risultati graditi ai bambini. Tra questi:

dei libretti dal testo breve, con le avventure di personaggi-chiave ("Petit Ours", "L'âne Tro-Tro", "La Chenille qui fait des trous", ecc) fotocopiati e poi colorati dai bambini. Abbiamo ritenuto utile ingrandire le pagine dei libretti e appenderle nello spazio allestito all'ingresso della scuola, così che i genitori potessero conoscere quanto veniva realizzato nelle sezioni e leggere le storie insieme ai propri figli;

le comptines;

i pictogrammes con i quali i bambini potevano comporre delle brevi frasi, imparando a strutturare il loro pensiero e arricchire progressivamente il loro vocabolario;

giochi vari come i domino e i memory realizzati dalle insegnanti, dipingendo le immagini su tavolette di legno compensato, adope- 


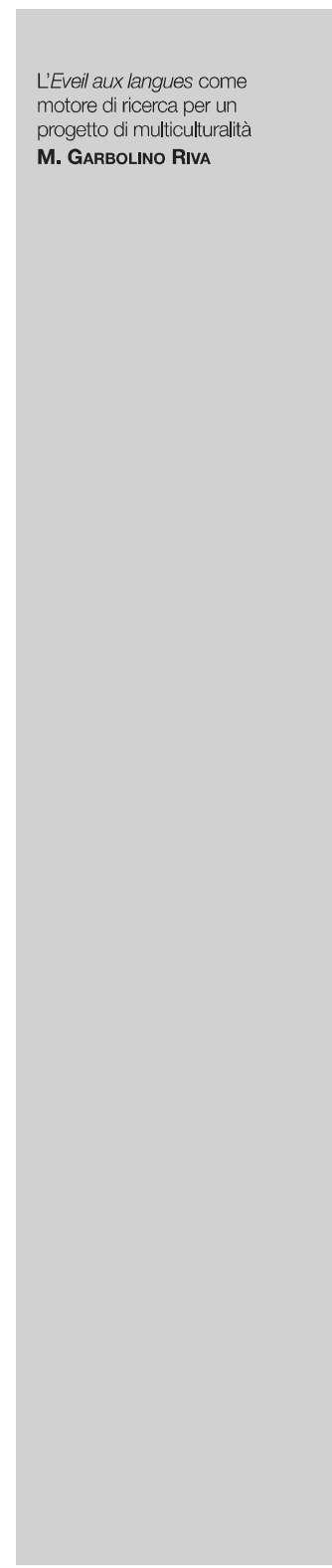

rati per giochi in piccoli gruppi di bambini, e finalizzati ad attività linguistiche e matematiche;

utilizzo di burattini, per mettere in scena dialoghi in lingua francese;

schede di lettura (immagini da commentare), per lo più collegate a temi stagionali;

alcune unità del metodo "Valentine et les autres". Realizzato all'inizio degli anni 80 e pubblicato nel 1983, il metodo ha segnato per la scuola valdostana il passaggio dall'insegnamento/apprendimento del francese, all'insegnamento/apprendimento in lingua francese. Esso è costituito da dodici unità didattiche formate da testi c immagini che hanno una progressione tematica e offrono occasioni per sviluppare attività psicomotorie, percettive, logiche, ritmico-musicali, grafiche e simboliche, utilizzando la lingua francese in molteplici esperienze espressive.

L'alternanza delle lingue italiana e francese nel corso delle attività didattiche, attraverso la modalità della mezza giornata da destinare a ciascuna, ha garantito l'integrazione delle due lingue e ha dato continuità al curricolo.

Per la lingua inglese la scelta metodologica è stata quella di affidare ad una collega competente l'organizzazione di un laboratorio di due ore settimanali per i bambini più grandi. L'insegnante ha seguito un programma così definito: imparare a salutare e congedarsi, presentarsi, nominare e riconoscere i principali oggetti scolastici, sapere riconoscere i colori, imparare a contare fino a dieci, memorizzare i nomi di alcuni animali, nominare i componenti principali della famiglia, conoscere le principali festività, identificare e riconoscere le differenti parti del corpo, associare dei vocaboli ai movimenti. Attraverso il gioco, le attività manuali, la coloritura di schede, l'ascolto di tracce audio, di canti mimati, e soprattutto attraverso la ripetuta condivisione con i compagni di quanto veniva proposto, i bambini hanno dimostrato di poter acquisire e mantenere nel tempo delle competenze linguistiche. Il materiale di ciascuno è stato raccolto in una cartellina personalizzata.

Un secondo progetto nel quale abbiamo utilizzato la lingua inglese è stato attivato in collaborazione con l'insegnante di motricità e ha avuto come destinatari i bambini di tre e quattro anni. Le esperienze motorie hanno favorito l'integrazione di diversi linguaggi, favorito la costruzione dell'immagine di sé e l'elaborazione dello schema corporeo in un contesto divertente, apprezzato dai bambini e favorevole per sensibilizzarli ad un codice linguistico diverso, che l'insegnante ha sempre utilizzato durante le sue lezioni. 


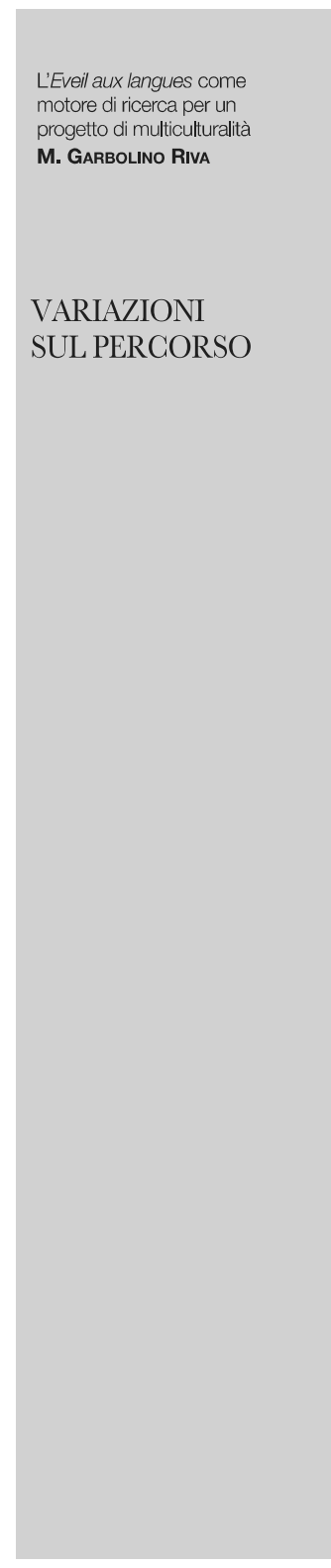

Quanto abbiamo descritto si riferisce alle iniziative che abbiamo messo in atto per realizzare il bilinguismo e l'inserimento della lingua inglese nel curricolo della scuola. Percepivamo tuttavia che rimaneva un po' in ombra un vero progetto di éveil aux langues, avente come obiettivo quello di promuovere la scoperta di altre lingue e delle relative culture. All'inizio dell'anno scolastico 2017/2018, il monitoraggio effettuato dalla Sovraintendenza presso le istituzioni scolastiche allo scopo di rilevare i dati della sperimentazione degli Adattamenti delle Indicazioni Nazionali alla peculiarità linguistica della Regione Valle d'Aosta, (come previsto dall'art. 39 dello Statuto), ci ha convinte a prendere in considerazione altri approcci per l'insegnamento bi-plurilingue, quelli che fanno ricorso a supporti innovativi quali i sacs à histoires, il kamishibaï, la boîte à histoires.

Per il loro corretto impiego abbiamo frequentato dei corsi di formazione organizzati dall'Amministrazione Regionale, così da potenziare le nostre competenze linguistiche e metodologiche. La conoscenza di questi strumenti adatti a promuovere il plurilinguismo ha dato corso ad un processo di ripensamento della nostra azione didattica, e nel contempo ci ha permesso di cogliere la presenza di una multiculturalità mobile e in crescendo che interessa anche la nostra scuola, poiché in essa sono presenti famiglie di culture diverse, europee ed extra europee. Verso la metà del mese di novembre 2017, abbiamo fatto un sondaggio per conoscere quali fossero le lingue parlate all'interno delle famiglie. Il risultato ci ha sorpreso: su un totale di 69 bambini frequentanti, le lingue familiari erano il russo (una famiglia), il portoghese (una famiglia), lo spagnolo (una famiglia), il tedesco (una famiglia), il rumeno (quattro famiglie), l'inglese (due famiglie) e l'arabo (una famiglia). A queste si doveva aggiungere il francoprovenzale (patois), una lingua minoritaria ma viva e presente nel contesto valdostano, più ancora del francese. Come fare emergere questa ricchezza nascosta e trasformarla in un patrimonio comune? Abbiamo iniziato gradualmente. Per preparare il cammino di Avvento abbiamo chiesto ad alcuni genitori di tradurre ventiquattro parole collegate al tema natalizio, in lingua tedesca, rumena, inglese, in francoprovenzale, oltre che naturalmente in lingua francese. Non abbiamo potuto inserire lo spagnolo e il portoghese, perché le famiglie interessate erano partite per le loro terre d'origine dove avrebbero trascorso le festività natalizie. Le prime parole plurilingui sono state quelle del Natale. Ogni mattina veniva letta ad alta voce la parola del giorno, rendendo protagonisti i bambini che conoscevano queste lingue e che diventavano i lettori della parola. I geni- 


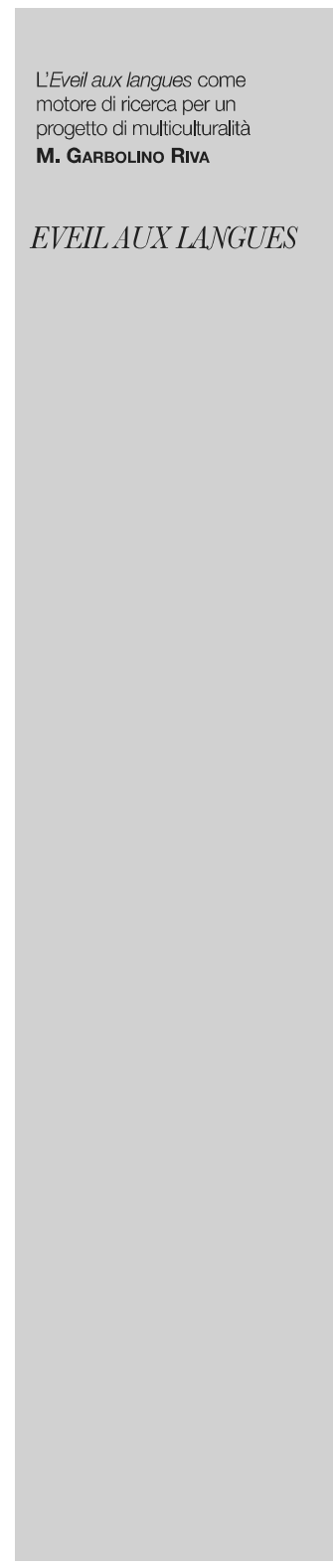

tori hanno seguito con interesse crescente questo processo di apertura della scuola al riconoscimento delle culture presenti in essa, che faceva emergere dall'invisibilità sociale le famiglie di origine straniera: un passo avanti nel processo di integrazione e di accoglienza di tutti, che ha allargato le maglie della rete sociale.

L'utilizzo del kamishibaï (che significa letteralmente "gioco teatrale di carte") è iniziato a fine novembre, e si è subito rivelato un originale ed efficace strumento per l'éveil aux langues. Si tratta di un teatro d'immagini di origine giapponese, anticamente utilizzato dai cantastorie ambulanti. In una valigetta di legno sono inserite delle tavole stampate su entrambe le facciate: da una parte il discgno e sul retro il testo e la numerazione della pagina. Mentre lo spettatore vede l'immagine, il narratore legge la storia facendo sfilare le tavole con le immagini salienti del racconto in questo teatro in miniatura chiamato butaï. Il Kamishibai invita a raccontare e a fare teatro, creando un forte coinvolgimento tra il narratore e il pubblico, esponendo i bambini all'ascolto e all'utilizzo di lingue diverse.

Parimenti iniziava anche l'utilizzo della boîte à histoires, uno strumento che permette di raccontare delle storie in diverse lingue, utilizzando oggetti che simbolizzano i personaggi e gli elementi chiave del racconto, e che l'insegnante fa uscire dalla scatola come per magia. $\grave{E}$ stato possibile raccontare delle storie nella lingua scelta dall'insegnante, integrandola con parole-chiave di un'altra lingua. Con modalità diverse, veniva ampliata l'offerta di contenuti rivolti alla curiosità dei bambini, i quali hanno risposto molto bene alle novità. Negli incontri di programmazione didattica abbiamo definito le scelte tecniche da mettere in atto, calendarizzando $\mathrm{i}$ temi da collegare con la programmazione annuale $\mathrm{e}$ chiedendo ai genitori che avevano collaborato con noi in tempo di Avvento di aiutarci per la traduzione e registrazione di alcuni testi nella loro lingua familiare. Tanto per il kamishibaï che per la boîte era infatti necessario avere a disposizione dei testi di racconti in più lingue, delle tavole disegnate e del materiale didattico finalizzato allo scopo. Ł̀ nata così una fruttuosa collaborazione, resa nota a tutte le famiglie dei bambini, che ha creato una realtà dinamica, con una molteplicità di soggetti in gioco. Alla ripresa della scuola dopo le vacanze natalizie abbiamo proposto ai bambini di diventare disegnatori di storie (a partire da quelle ascoltate insieme), da inserire nel loro personale, piccolo kamishibaï costruito con il cartoncino ondulato. La proposta è stata accolta con interesse e, superate le difficoltà tecniche rappresentate dallo scor- 


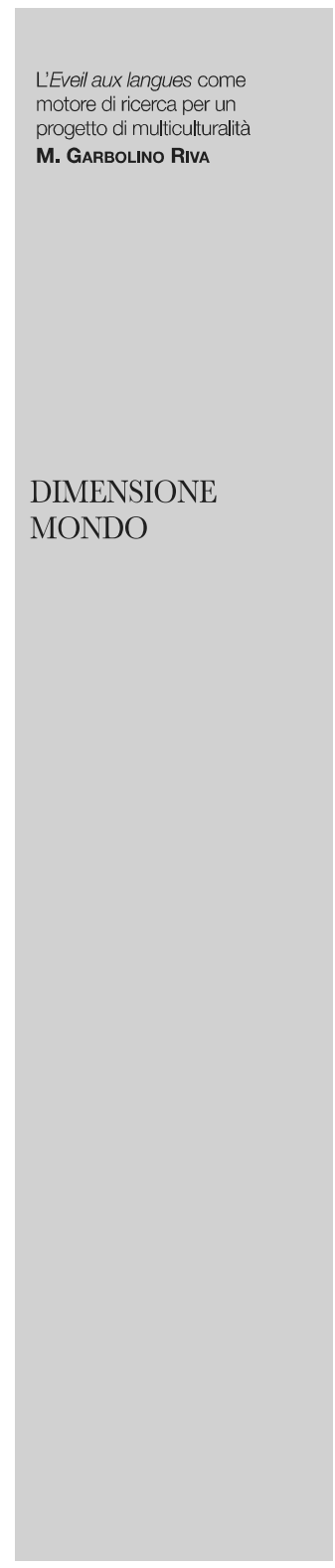

rimento delle immagini del butai, ogni bambino a metà febbraio ha portato a casa questo oggetto con la storia da narrare, da lui stesso disegnata. I bambini di quattro anni hanno incontrato qualche difficoltà nella ricostruzione delle sequenze temporali, pertanto abbiamo consegnato loro i disegni già numerati sul retro e pronti da colorare. Otto sono i racconti realizzati a scuola, frutto della collaborazione tra insegnanti e genitori, con l'utilizzo della lingua italiana, francese, inglese, ma anche con l'inserimento del portoghese e del russo. Per l'ascolto dei testi nelle lingue straniere ci siamo avvalse delle registrazioni su CD fatte dai genitori di madre lingua. Quattro sono invece i testi per la boîte à histoires, tratti dalle fiabc più note della letteratura infantilc.

La scuola rappresenta, all'interno della società multietnica che si sta delineando, un ambito in cui la multiculturalità acquista immediata visibilità e un ambito privilegiato e strategico per la costruzione di interculturalità. Da questa convinzione è scaturita la necessità di aiutare $\mathrm{i}$ bambini a comprendere da dove vengono o dove vivono le persone che parlano lingue diverse dall'italiano, strutturando delle unità didattiche aventi come tema: in viaggio intorno al mondo. L'éveil aux langues diventava così il motore di ricerca, il volano per un progetto più ampio, che aveva l'ambizione di aprirsi alla conoscenza di altre culture e universi linguistici, come indicato nel titolo che abbiamo dato a questa ricostruzione del nostro percorso didattico. Abbiamo informato le famiglie degli scopi del progetto, chiedendo la loro collaborazione in una serie di iniziative di sostegno, e allestito nell'atrio della scuola uno spazio espositivo dove raccogliere gli elementi principali dell'ambiente che di volta in volta era oggetto della nostra visita di turisti curiosi. Ogni bambino ha preparato il suo personale passaporto (un pieghevole prestampato) personalizzandolo con il disegno del proprio volto e con la scritta del proprio nome in stampatello (o con una propria sigla riconoscibile); in un apposito spazio bianco veniva apposto un timbro di convalida ad ogni tappa del viaggio. Di ognuno degli Stati visitati è stata fatta una presentazione generale, cercando fotografie, immagini, ricostruendo in cartoncino ondulato i principali monumenti per poi collocarli in un plastico, evocando aspetti legati alla cultura, allo stile di vita, ai linguaggi propri del luogo. Il progetto di ricerca è stato un work in progress che si è ampliato ben oltre le aspettative iniziali. I modellini delle piramidi egiziane, le navi dei conquistatori e dei pirati caraibici, i villaggi indiani si sono alternati con gli igloo del continente artico, con i paesaggi messicani e peruviani, con la foresta amazzonica e 


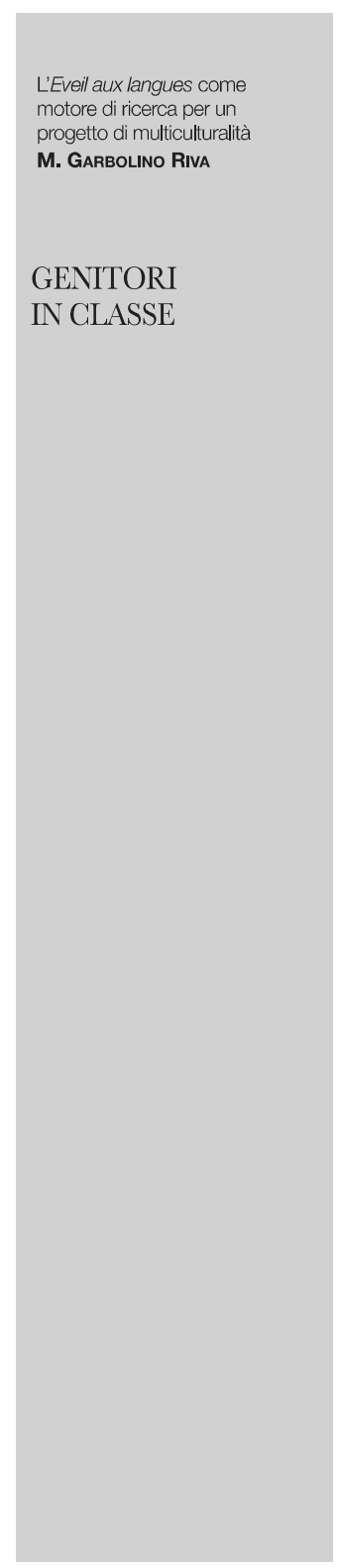

la spiaggia di Copacabana, con le pagode e la muraglia cinese, le isbe russe, i mulini dell'Olanda, i monumenti di Parigi e di Londra. All'aspetto ludico, per certi aspetti trainante agli occhi dei bambini, poiché li ha resi attivi e propositivi, abbiamo sempre tenuto congiunto l'obiettivo linguistico/culturale che è stato realizzato con il contributo importante di alcuni genitori.

Nella tappa in cui abbiamo raggiunto il continente latino americano abbiamo chiesto a due mamme di bambini di nazionalità peruviana e brasiliana di venire a scuola per presentare la loro terra di origine, portando qualche oggetto caratteristico, così che il supporto delle immagini tratte dai libri fosse arricchito c completato dall'incontro con un testimonial d'eccezione quale può essere solo un genitore. La loro presenza ha elevato la qualità del progetto, autenticandone la validità dal punto di vista culturale, e ha offerto alla comunità scolastica la risorsa impagabile di una persona portatrice di una propria lingua familiare: in questo caso lo spagnolo (lingua nazionale del Perù) e il portoghese (nella versione utilizzata in Brasile). Per ognuna di queste due lingue i bambini hanno ascoltato e appreso qualcosa: la numerazione da uno a dieci in spagnolo, i colori in lingua portoghese e una facile canzoncina da mimare.

Volendo evitare che la partecipazione diretta dei genitori in classe si risolvesse in un avvenimento estemporaneo per quanto simpatico, (poiché le mamme hanno fatto a gara per offrire un piccolo dono a tutti: un sacchetto di ceci di importazione peruviana, una bandierina brasiliana), ma senza un diretto collegamento con il percorso didattico, al termine di ogni singola tappa del viaggio abbiamo cercato di proporre un'attività tesa a verificare l'efficacia dell'esperienza vissuta. Ecco alcuni esempi: il gioco dell'oca in cui si avanza nella casella successiva rispondendo alla domanda con una parola appresa in una determinata lingua (il nome di un colore in portoghese, di un animale in inglese, un numero in francese, ecc); la mappa del tesoro con i numeri in lingua spagnola; la realizzazione di una maschera africana in rafia colorata, abbinata al testo di una canzoncina in lingala; i saluti scritti nelle diverse lingue su cartoncini colorati, ripresi nel gioco del memory. Le tavole del kamishibaï relative al mese di marzo sono state dedicate alla storia del pesciolino "Arc-en-ciel" che i bambini hanno ascoltato in italiano e portoghese. Mantenendo un forte collegamento con l'aspetto ludico, l'esperienza ha conservato il fascino della sensibilizzazione alle diverse sonorità linguistiche, senza diventare un insegnamento forzato. Anche per il nostro viaggio virtuale in Russia e 


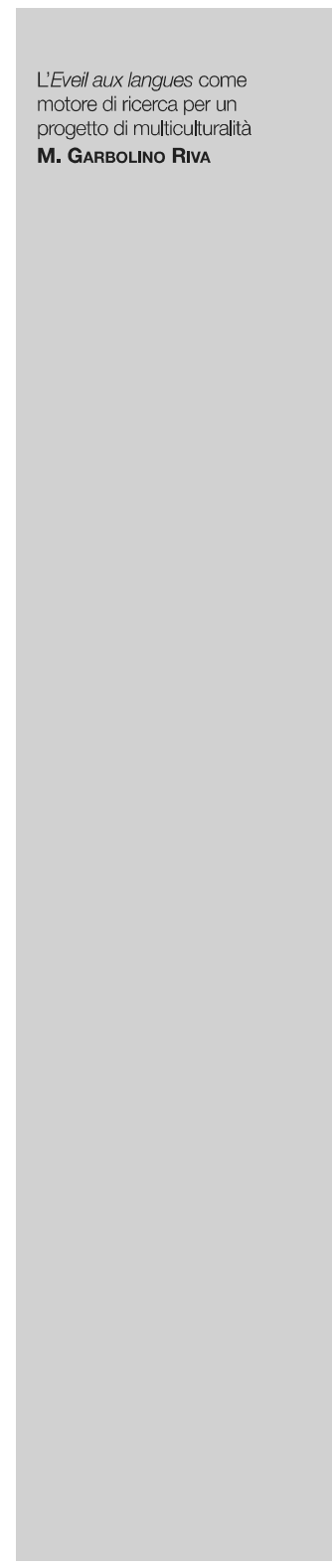

Romania abbiamo invitato a scuola due genitori che hanno intrattenuto i bambini mostrando oggetti e giocattoli caratteristici della loro cultura d'origine. In sezione i bambini hanno realizzato in cartoncino cinque matrioske di grandezza diversa, divertendosi poi a disporle in ordine crescente o decrescente. Qualcuno è riuscito ad imparare a contare fino a 5 in russo, ascoltando ripetutamente la registrazione della voce di un loro compagno di scuola. Questa conoscenza numerica è stata inserita nel racconto "Kim le pingouin", presentato con il kamishibaï.

Dalla tradizione rumena abbiamo invece attinto la preparazione di un mărtişor, un piccolo pupazzetto in lana rossa e bianca, usato prevalentemente dalle ragazze per festeggiare l'inizio della primavera, e la decorazione delle uova rosse. Da alcuni anni la comunità romena costituisce la componente più rilevante della nostra scuola; attualmente sono quattro famiglie, molto solidali tra loro e determinate nel mantenimento delle proprie tradizioni, sia civili che religiose. Nei giorni precedenti la Pasqua ci hanno offerto un assaggio dei dolci tipici della loro tradizione e un bel libro sulle icone ortodosse.

Il percorso iniziato a gennaio si è concluso a maggio con l'ultima tappa dedicata alla regione Valle d'Aosta e alla lingua francoprovenzale, il patois. Questa lingua è a tutt'oggi l'elemento identitario più forte, il filo rosso che unisce le comunità che si sono insediate attorno al Monte Bianco. Spesso il termine patois è diventato sinonimo di dialetto, anche se si tratta di una vera e propria lingua con caratteristiche specifiche che l'Amministrazione Regionale valorizza mediante iniziative destinate alle scuole.

Anche per la presentazione di questa lingua locale ci siamo avvalse dell'aiuto di alcuni genitori, sia per gli aspetti linguistici, sia per la documentazione e per i materiali che ci hanno fornito: registrazioni di canti in patois, ricerca degli strumenti musicali e dei costumi tipici dei gruppi folcloristici valdostani. Tutto ha contribuito a rendere tangibili alcuni aspetti della cultura alpina, sconosciuti ai bambini che oggi vivono in un contesto prevalentemente cittadino.

Il percorso ora descritto, che potremmo definire di progressione a spirale, ci ha permesso di osservare i bambini nel loro contesto di apprendimento-sperimentazione nei diversi campi di esperienza, e di raccogliere degli elementi utili per la compilazione della scheda informativa regionale di descrizione delle competenze raggiunte dagli alunni. Infatti tra i descrittori contenuti nel profilo del bambino in uscita dalla scuola dell'infanzia ve ne sono alcuni che fanno riferimento all'acquisita consapevolezza dell' esistenza di 


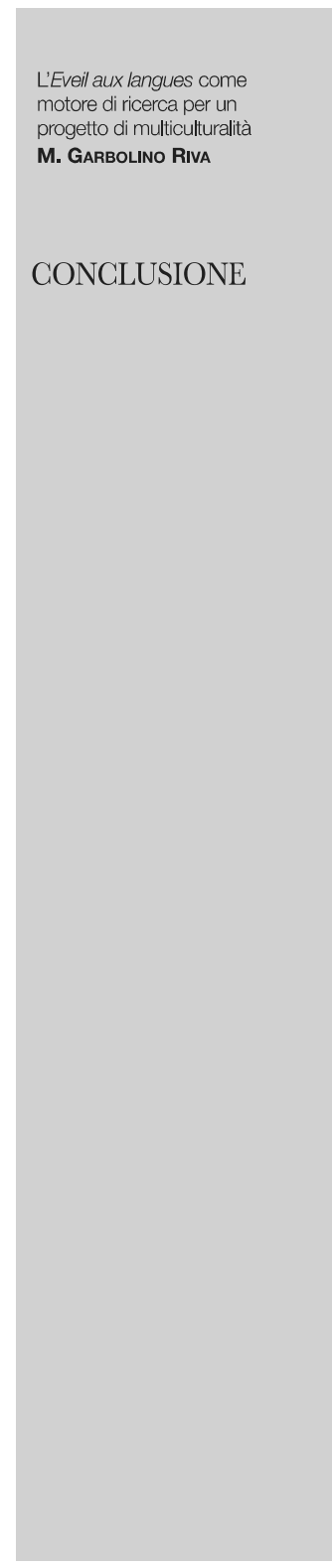

codici linguistici diversi da quelli familiari, della presenza di una pluralità di lingue nel mondo e di una diversità di universi sonori. Questi obiettivi di sensibilizzazione alle lingue si possono raggiungere anche con un numero ridotto di lingue presenti nel proprio contesto scolastico, se si fa ricorso a strumenti metodologici adeguati.

La distanza che separa quest'ultima pagina dalla premessa scritta nella prima è pari ad un intero anno scolastico, ma ora è possibile riprendere la domanda di partenza e offrire una risposta motivata. L'éveil aux langues si è davvero dimostrato un motore di ricerca adeguato non solo per conoscere le diversità linguistiche c culturali, ma anche per fare della lingua di ciascuno un'opportunità di conoscenza per tutti all'interno della scuola. Analizzando le esperienze compiute in questi mesi, e di cui abbiamo dato ampio resoconto, rimane da dire ancora qualcosa sull'impatto che tutto questo ha avuto sulle famiglie. Quelle maggiormente coinvolte nel progetto ci hanno più volte attestato la loro gratitudine per l'attenzione a loro rivolta e per la valorizzazione della loro cultura; per le altre famiglie, si è trattato di scoprire insieme ai loro figli gli aspetti comuni a tutte le culture, a partire dalle forme più semplici. Ogni cultura conserva temi ricorrenti, simili, o che rimandano alle altre: il saluto, il momento del pasto, il vestiario, il tempo che prepara alla nanna, il corpo, gli animali, le feste. Gli stessi genitori ci hanno fatto notare come spesso ad una canzoncina ne corrisponda un'altra, in una lingua diversa, che evoca lo stesso soggetto. È dunque possibile costruire dei legami tra una lingua e l'altra, cogliere le somiglianze, abituare l'orecchio alle molteplici sonorità linguistiche (Frère facques è un canto che si ritrova in molte lingue). Anche i saluti nelle diverse lingue, i canti abbinati alla gestualità, hanno contribuito a creare dei legami tra le persone di culture diverse presenti a scuola o quanto meno a metterle in evidenza e in contatto tra loro. Si conferma come attraverso il prisma della lingua venga favorito un modo positivo di concepire il vivere insieme, in una società che diventa sempre più multietnica $e$ come gli obiettivi individuati per i bambini possano declinarsi anche per gli adulti, educandoci ad una cittadinanza che sia rispettosa delle differenze. Come diceva Federico Fellini: "Un linguaggio diverso è una diversa visione della vita". I bambini non hanno imparato altre lingue, ma attraverso un approccio ludico hanno acquisito la capacità di creare dei legami tra differenti modi di esprimersi. Questa competenza sarà riattivata nella scuola primaria e sviluppata nel corso dell'apprendimento della lettu- 


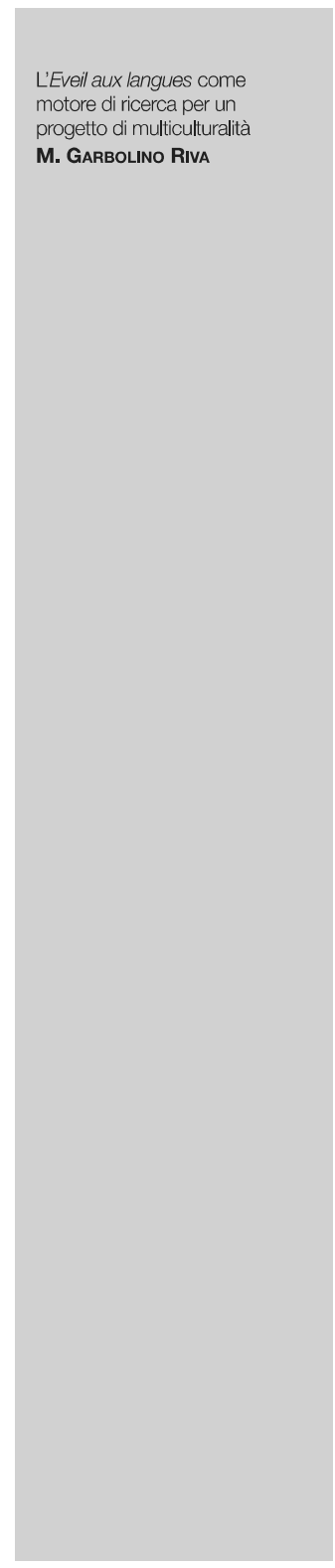

ra e della scrittura, quando essi assoceranno suoni a grafemi.

Un'ultima breve nota riguarda la terminologia utilizzata per spiegare alle famiglie il nostro progetto di éveil aux langues. Lo abbiamo sempre descritto come un tentativo di mettere al centro le lingue familiari, evitando la stigmatizzazione di lingua straniera, che sottolinea la non appartenenza al gruppo. Ci sembrava importante de-costruire terminologie che per abitudine perdurano nelle nostre pratiche, e che sottolineano il sistema di rappresentazione identitaria tra "noi" e "loro", soprattutto da parte della cultura maggioritaria presente a scuola. Entrare nella cultura/lingua ha richiesto una certa empatia e una conversione pedagogica ad iniziare da noi, registi c strumenti di un progetto di ricerca che ha colmato un anno di lavoro; vogliamo sperare che tutto ciò abbia riflessi positivi nella vita dei bambini, adulti di domani in una società dove la conoscenza delle lingue avrà un ruolo fondamentale. $\grave{\mathrm{E}}$ nostro convincimento che resti sempre attuale quanto ha scritto don Lorenzo Milani: "Il maestro deve essere per quanto può, profeta, scrutare i segni dei tempi, indovinare negli occhi dei ragazzi le cose belle che essi vedranno chiare domani e che noi vediamo solo in confuso". 